\section{Regional variations in US cancer imaging data: a warning for imaging overuse}

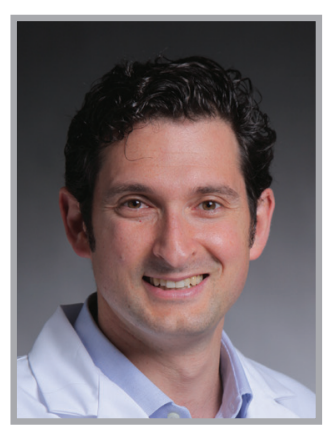

Danil V Makarov* speaks to Gemma Westcott, Commissioning Editor: Danil V Makarov is an Assistant Professor and Director of Surgical Research in the Department of Urology at NYU Langone Medical Center (NY, USA). In addition, he is an Assistant Professor in the Department of Population Health. His clinical areas of expertise include prostate cancer, benign prostatic hyperplasia, erectile dysfunction, kidney cancer, urinary tract infections, genitourinary neoplasm, elevated prostatespecific antigen and testicular cancer. In addition, his research interests are in the areas of prostate cancer, health policy and quality of care. An alumnus of the Johns Hopkins University School of Medicine (MD, USA), he completed his residency in urology at Johns Hopkins Hospital and a research fellowship at Yale University School of Medicine (CT, USA).

\section{Q Can you tell our readers about your career to date?}

I am a urologist and a health services researcher, and I take care of patients with prostate cancer and other urological malignancies clinically. I spend half of my time doing that and I spend the other half of my time doing health services research, which is the intersection between health policy, epidemiology and healthcare economics. I am particularly interested in the cost and quality of care for men with prostate cancer and how those are affected by changes in healthcare policy and the diffusion of new practices and innovations.

\section{Q What initially sparked your interest in cancer research?}

This dates back to medical school. I was always interested in cancer in general and particularly in prostate cancer; I was fascinated with issues surrounding screening, diagnosis and treatment as a medical student. On my rotations I got to meet different providers and I felt like I really fitted in and got on well with the urologists. I got encouragement when I was at medical school from some great mentors at John Hopkins and I ended up staying there for a residency. There, I did a lot of work in clinical epidemiology and risk stratification of men with prostate cancer, but I was always drawn to the notion that as good as prediction models are, they are never perfect. There was so much wrong in the way that healthcare was delivered that if we could just get people to do basic things correctly, we would do as much or maybe more for patients than discovering a whole lot of new things. It is well known how long it takes for a discovery to be made before it is implemented into clinical practice. From there, I did a fellowship that was sponsored by the US Department of Veteran Affairs Veteran Association (VA) and The Robert Wood Johnson Foundation and I got to work with a wonderful mentor there Harlan Krumholz and another one, Cary Gross who nurtured my thinking along these lines and here I am today.

*Assistant Professor \& Director of Surgical Research, Departments of Urology \& Population Health, Member of the Laura \& Isaac Perlmutter Cancer Center, NYU Langone Medical Center, Staff Surgeon, VA New York Harbor Healthcare System, NY, USA

\section{Future ONCOLOGY}

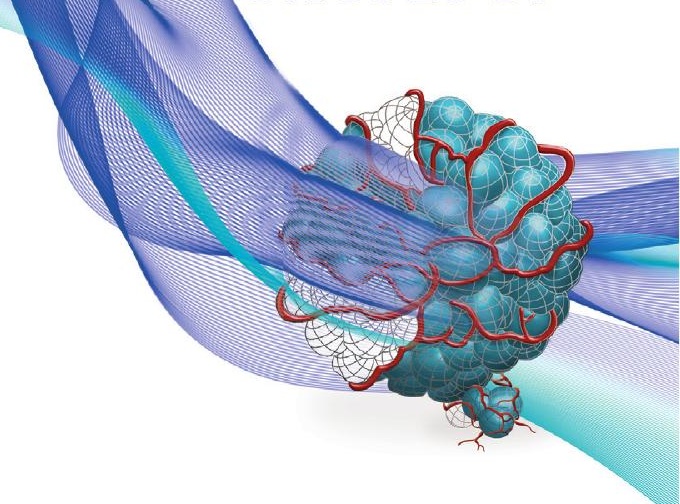

\section{KEYWORDS}

- breast cancer $\bullet$ cancer $\bullet$ diagnostics

- healthcare $\bullet$ healthcare

systems/services $\bullet$ information

management/tracking systems

- insurance $\bullet$ medicine/health

- prostate cancer $\bullet$ public health
Future Medicine 
Q You recently published a paper regarding regional variations in prostate $\&$ breast cancer imaging use in America. Can you tell us about the study \& what you found?

In the USA and around the world, for a very long time we have known that there are variations in practice of care when you look across various regions or physicians at any level. I think, in the USA, recent policy efforts to correct that have focused on the individual interactions between patient and physician, so there are a lot of efforts to optimize that interaction with decision support and shared decision making and trying to make sure that every time the physician and patient meets, they always come up with the best solution. I love that. I think those are tremendous and important policy efforts and they are critical. However what I have found in some of my research is that it appears that a lot of regional variation appears to be driven not necessarily just by randomness or variation in these individual interactions, but there appear to be regional-level issues and regional-level factors that are responsible for a lot of this variation.

We have done a lot of work in prostate cancer and have found a lot of suggestion that there are regional differences in propensities for imaging. In certain regions, the physicians use a lot of imaging all of the time and other regions they use imaging a lot less. We thought that if indeed there were regional level factors that were driving imaging for prostate cancer, would those same regional factors be driving imaging in other states? We chose breast cancer, which is a disease which affects a completely different group of patients, as prostate cancer exclusively affects men while breast cancer almost exclusively affects women. Often, it is very different providers that care for prostate cancer of women with breast cancer. People tend to specialize in one or the other. If all of these decisions were random variation within the providers, you would assume that there would be no association between prostate and breast cancer imaging at a regional level. However, we found that there was an association, which suggests that there are practice-norms or a kind of medical culture in these regions that influence physicians and patients within certain wands to be imaging-avid and to use a lot of imaging.

\section{Q How did you conduct the retrospective study?}

We use the SEER-Medicare Linked Database. The National Cancer Institute (NCI) collects cancer data in the USA through a program through SEER (which stands for Surveillance Epidemiology End Results) and it has 17 registries which collect all of the information about cancer cases and diagnosis and initial treatment within their registries. Within these registries, when patients are diagnosed with cancer it is reported back to the NCI and we consider the cases to be representative of population-level data. The NCI only collect on cancer incidence and cancer characteristics at first treatment and they do not collect information on other health services patients may use; however, for patients over 65 years we have Medicare, which is a statesponsored program that provides healthcare subsidies and is linked to SEER. You therefore have not only clinical data about the patients, but also data about what healthcare resources they have used. Using that, we were able to determine cancer-grade stage prostate-specific antigen for men with prostate cancer and we were able to look for billing codes for imaging. Based on the clinical settings, we could classify them as 'needing imaging' or not 'needing imaging' based on the clinical oncology recommendations for who does not need imaging specifically for this study. Then we could look at the Medicare portion of the database to see if they had the imaging or not. That is how we built our dataset and evaluated our end point.

\section{Q What harm can imaging overuse bring to patients \& clinical practice?}

Wasteful medical spending is harmful; it is money that is spent on a procedure that does not need to be done and does not provide any useful information. Metastatic or advanced disease is so rare in patients that have the clinical characteristics that we have defined in this study and that groups like the American Society of Clinical Oncology have defined as low-risk, that it is incredibly unlikely to find something that will change the treatment or management strategy for patients, so it is much more likely to be a false positive than a true positive if you have a positive study. Therefore, you have wasted the money to do the test in the first place, you have taken time out the patient's schedule and then there is the anxiety that comes with that too. If you do get a positive, even though it is very likely to be a false positive, once you have something abnormal, it is going to require further follow-up and that can take the form of invasive testing, which can cause discomfort, or it can 
require more imaging which costs more money and wastes more time. In the end, it is not going to do much to guide treatment. It is not as if people are dying of these excess scans but it is a wasteful effort all round.

\section{Q Can you tell us about Choosing Wisely} $\&$ what implications these findings have on the campaign?

Choosing Wisely is a joint effort of the America Board of Internal Medicine and Consumer Reports. It is a campaign which enlists specialist societies to identify practices which when decreased could both improve care and save money, to ultimately encourage stewardship of healthcare resources. A number of speciality societies have participated and two specialist societies, The American Urological Association, of which I am a member, and the American Society of Clinical Oncology, have both named prostate cancer imaging among low-risk patients as a priority in their statements identifying the top five practices which could be eliminated to save money and improve care. The American Society of Clinical Oncology actually named prostate cancer and breast cancer, which was very fortuitous for us, again because I was describing this nonoverlapping group of patients. Choosing Wisely, which I do think is a really great initiative, puts the onus of discussion on the individual physician and patient. They are meant to discuss these things if they are relevant. I think it is a great idea, however in some of our research that we are conducting now when we are talking to physicians and patients, none of the patients have heard of Choosing Wisely and few of the physicians really know what it is. Unfortunately, it is not being embraced in routine clinical practice as much as it is in the academic and health policy communities.

\section{Q Does this research support population-based decision-making?}

I think that individual and shared decisionmaking and improving that individual decision making is vital and critical and needs to continue. I am not advocating for one versus the other, I am advocating for one and the other. We need to continue to Choosing Wisely and shared decision-making. We need to continue decision support to optimize decisions at the individual level. What we can conclude - even though what we found is hypothesis-generating at this point - is that because of the potential of the existence of these regional level factors, it is worthwhile to study them more to understand them better, so we can craft policies that can work at the system level. I think there will be a role for both - I hope!

\section{Q How can policy-makers \& researchers use your findings to promote appropriate cancer care?}

In terms of direct practice right now, when you see these sorts of data and you see the vast variation in appropriate imaging use and the rates of very high imaging use, I would hope that it would make people pause and consider how often they are doing it in their own practice. In terms of concrete, novel policy approaches based on this, I think we are not ready for prime-time yet. I think this is research that is hypothesisgenerating that will hopefully stimulate more research so that we can understand what these factors are. Once we have understood what the factors are, then we should craft policy solutions based on them. I think hastily conceived policy solutions can be fraught with unintended consequences.

\section{Q What is next for your research?}

Right now, we are working on characterizing the barriers to implementation of imaging guidelines within the VA, which is committed to providing quality care, research and teaching and they fund our research. We are looking at the causes of inappropriate imaging use in the VA and we are getting some handle on why that is. We are working on designing an implementation effort to decrease inappropriate prostate cancer imaging. I think with this finding that comes along, it suggests that there are regional-level factors, but for me I would like to branch out and look at more types of cancers and other types of diseases to see if there are any commonalities that we can address in these policy efforts - those are the next steps for me.

Q Finally, where would you like to see cancer imaging in the USA \& around the world in 10 years' time?

In 10 years' time, I would like cancer imaging to be much more guideline-concordant. I think we have a lot of great guidelines out there by a number of different policy organizations. Certainly for prostate cancer and for breast cancer there is a really good consensus among 
a lot of organizations that there is a right way to be doing this. My hope for the future is that there will be more decision-support for physicians through electronic medical records and through conferences among physicians where they can tease out when it is appropriate and not appropriate to use these tests. I would hope that we could move toward a better understanding of the guidelines and move closer to the guidelines in practice, but we should do this rationally so that not every single person has to do it or else, but that people understand it. If we veer off, it is for a good reason and it should be driven by the patients and patient values rather than by the physician's values.

\section{Disclaimer}

The opinions expressed in this interview are those of the interviewee and do not necessarily reflect the views of Future Medicine Ltd.

\section{Financial \& competing interests disclosure}

DV Makarov has no relevant affiliations or financial involvement with any organization or entity with a financial interest in or financial conflict with the subject matter or materials discussed in the manuscript. This includes employment, consultancies, honoraria, stock ownership or options, expert testimony, grants or patents received or pending, or royalties.

No writing assistance was utilized in the production of this manuscript. 